\title{
Génération de rayonnements UV et VUV par décharge de surface
}

\author{
M.L. Sentis, F. Chazaud, H. Rigneault, W.I. Marine* et A. Paramonov** \\ Institut de Mécanique des Fluides de Marseille, URA 783 du CNRS, Campus de Luminy, \\ case 918,13288 Marseille cedex 9 , France \\ * Groupe de Physique des Etats Condensés, URA 783 du CNRS, Campus de Luminy, \\ case 918, 13288 Marseille cedex 9, France \\ ** Lebedev Institut of Physic, Académie des Sciences de Russie, 53 Leninsci Avenue, \\ Moscou 117924, Russie
}

\begin{abstract}
Résumé : L'hydrodynamique et les propriétés spectroscopiques d'un plasma créé par décharges de surface sur ferrites sont étudiées. Le domaine spectral considéré est celui de l'ultraviolet du vide. Les décharges électriques (200 à $1000 \mathrm{~J}$ ) réalisées dans de l'argon ou de l'hélium (10-4 Torr à 1,5 bar) conduisent à la formation d'un plasma de forme demi-cylindrique et d'une onde de choc. Nous montrons que leur propagation spatio-temporelle dépend de l'énergie injectée et de la densité du gaz. Les émissions intenses observées entre $115 \mathrm{~nm}$ et $220 \mathrm{~nm}$ sont attribuées aux particules éjectées de la surface du matériau, à leur interaction avec le gaz environnant, et à l'injection directement dans le gaz, d'une partie de l'énergie électrique stockée.
\end{abstract}

\begin{abstract}
Dynamic and spectroscopic characteristics of a vacuum ultraviolet formed ferrite plasma source are presented. Electrical discharges (200 à $1000 \mathrm{~J}$ ) in argon or helium (10-4 Torr to 1,5 bar) lead to the formation of a halfcylindrical plasma column surrounded by a layer of shock-compressed gas. We show that the plasma and shock wave propagation depends on the deposited energy and on the background gas density. Spectroscopic study in the 115-210 nm spectral region has been carried out. Emission spectra are dominated either by atomic lines superimposed on continuum emission depending on the surrounding gas. Atomic lines are related to the emission from electronic transitions of singly or multi-ionized atomic species ejected from the ferrite.
\end{abstract}

\section{INTRODUCTION}

Les décharges de surface sur polymères, céramiques, ou autres matériaux diélectriques permettent l'obtention d'émissions lumineuses incohérentes impulsionnelles de forte puissance. Ces sources lumineuses, dont le principe de fonctionnement et la mise en oeuvre sont simples, sont généralement caractérisées par une émission large bande dans les domaines spectraux du visible et de l'ultraviolet. Les décharges électriques sur la surface de ferrites $\left(\mathrm{Ni}-\mathrm{Zn} \mathrm{Fe}_{2} \mathrm{O}_{4}\right.$ ) font partie de ces sources; elles sont plus généralement connues sous le générique "Formed-Ferrite Plasma source" ou plus 
simplement "FFP source"1. Leur principale originalité est la possibilité d'obtenir des émissions intenses principalement dans les domaines de l'ultraviolet (UV), de l'ultraviolet du vide (VUV) ${ }^{2}$ et des rayons $X^{3}$. La durée de l'émission incohérente varie généralement d'une microseconde à une dizaine de microsecondes selon la nature et la pression du gaz environnant, la géométrie de la décharge de surface et le type de circuit d'excitation électrique. Ces sources peuvent également fonctionner à des fréquences de répétition relativement élevées $(\sim 100 \mathrm{~Hz})$ avec une durée de vie élevée $\left(\sim 10^{5} \text { à } 10^{6} \text { tirs }\right)^{4}$. Les domaines d'applications des sources VUV sont essentiellement, la micro-électronique, le nettoyage de surface, la préionisation et le pompage optique de lasers, et la photochimie ${ }^{5}$.

Après une description sommaire du dispositif expérimental, une étude spatiotemporelle de la formation et de l'expansion du plasma à la surface d'un barreau de ferrite est présentée. Les principaux paramètres étudiés sont la nature du gaz environnant les ferrites $(\mathrm{He}, \mathrm{Ar})$ et l'énergie déposée à la surface de celles-ci (de 0,2 à $1 \mathrm{~kJ})$. Les résultats expérimentaux issus de l'étude spectroscopique du plasma, dans un domaine spectral compris entre $115 \mathrm{~nm}$ et $220 \mathrm{~nm}$ sont également présentés.

\section{DISPOSITIF EXPERIMENTAL}

La figure 1 est une représentation schématique du dispositif expérimental. Celuici ayant été décrit en détail précédemment 6 , une présentation sommaire sera donnée ici. La cellule d'essais d'un volume de 4,5 litres $\left(40 \times 20 \times 5,6 \mathrm{~cm}^{3}\right)$ permet la fixation entre deux électrodes d'un ou deux barreaux de ferrites de $1 \mathrm{~cm}$ de diamètre et d'une longueur de $20 \mathrm{~cm}$. Deux fenêtres en $\mathrm{MgF}_{2}$ sont placées de part et d'autre de la cellule pour permettre une visualisation transversale du plasma. Enfin, la pression à l'intérieur de l'enceinte peut varier de $10^{-4}$ Torr à 1,5 bar.

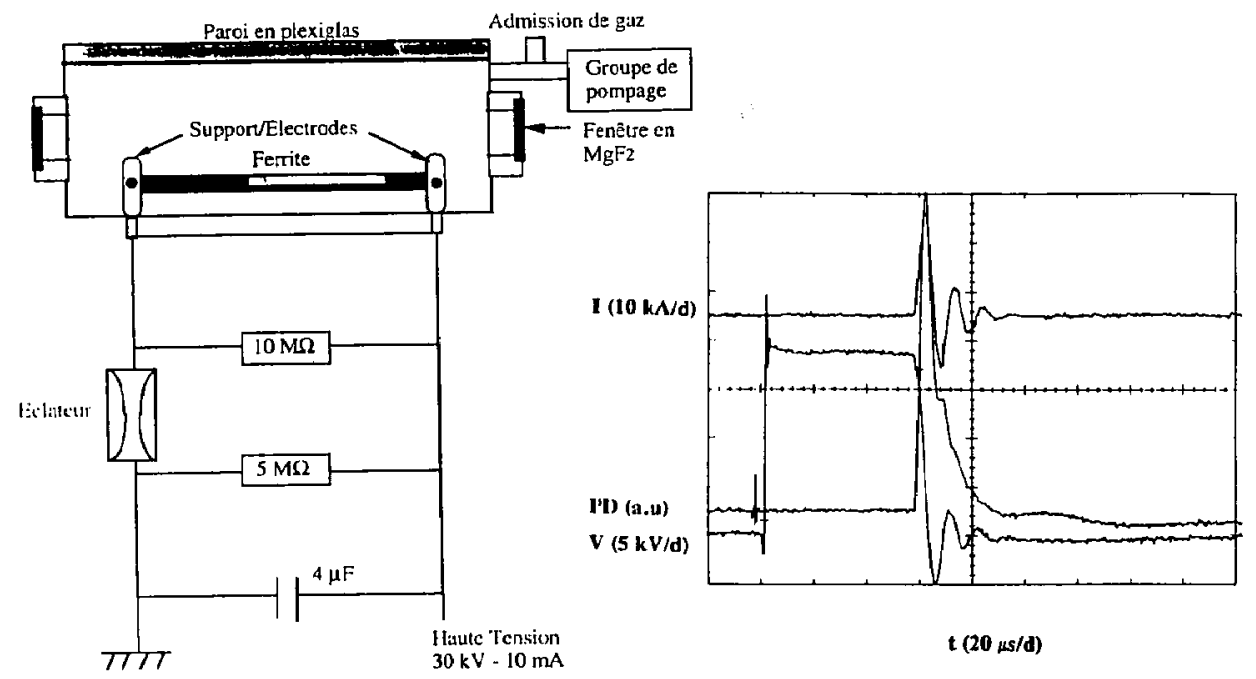

Fig. 1 : Schéma du dispositif expérimental.

Fig. 2 : Evolution temporelle du coturant (I), de la tension $(V)$ et du signal lumineux (PD). 
Le circuit électrique, d'une très grande simplicité, est constitué principalement d'un condensateur de stockage de $4 \mu \mathrm{F}$ chargé à une tension maximale de $30 \mathrm{kV}$ et d'un éclateur pressurisé à l'azote.

Les ferrites utilisées, de type $(\mathrm{Ni}-\mathrm{Zn}) \mathrm{Fe}_{2} \mathrm{O}_{4}$, sont des matériaux microcristallins frittés présentant une résistivité très élevée $\left(10^{8} \Omega . \mathrm{cm}\right)$. L'initiation d'une décharge de surface sur ferrite avec une valeur raisonnable du seuil de tension de claquage de la décharge $(0,1$ à $1 \mathrm{kV} / \mathrm{cm})$, nécessite une diminution notable de la résistivité du matériau. Par ailleurs, l'obtention d'un rayonnement intense de courte longueur d'onde requiert un confinement de l'énergie injectée à la surface du matériau. Ces deux conditions sont obtenues dans notre cas par la formation d'un "canal conducteur" sur la surface de la ferrite. L'étude de la formation de ce canal a fait l'objet de précédents travaux ${ }^{6}$. La diminution de la résistivité dans le canal conducteur de quatre à cinq ordres de grandeur a été obtenue par irradiation laser infrarouge ou par l'utilisation d'un fil explosif. Les canaux ainsi réalisés ont une largeur comprise entre 0,1 à $1 \mathrm{~mm}$ de large. La chute de la résistivité s'explique par la modifcation de la structure du réseau cristallin du matériau et par la formation en surface d'oxyde de fer, composé bon conducteur.

La figure 2 montre l'évolution temporelle du courant (I) et de la tension (V) de la décharge ainsi que l'émission lumineuse dans le domaine du visible et de l'ultraviolet mesurée à l'aide d'une photodiode. La tension de charge est de $19 \mathrm{kV}$ et la pression d'argon est égale à 1 bar. Après le déclenchement de l'éclateur, la tension aux bornes des électrodes atteint très rapidement une valeur proche de celle de la tension de charge. Cette tension se maintient pendant une durée d'environ $60 \mu \mathrm{s}$ avant de chuter brutalement. La durée de ce palier est peu dépendante de la nature du gaz environnant mais dépend fortement de la distance inter-électrode et de la résistivité initiale du canal conducteur. Durant cette étape un très faible courant (de un à quelques dizaines d'Ampère) traverse le canal conducteur, il suffit cependant à faire chuter l'impédance du canal par chauffage ohmique. La décharge s'établit alors avec l'apparition d'un fort courant et la formation du plasma, puis la tension et le courant oscillent avec une fréquence dépendante de l'accord d'impédance entre le circuit d'excitation électrique et le plasma. L'impédance du plasma varie fortement selon la nature du gaz et la longueur de la décharge, et dépend faiblement de la résistivité initiale du canal conducteur.

\section{HYDRODYNAMIQUE DU PLASMA}

L'étude de l'hydrodynamique du plasma et de l'onde de choc créés par la décharge permet une meilleure compréhension des processus physiques mis en jeu lors de la décharge. Elle conduit aussi à la détermination de la forme géométrique du plasma et de sa vitesse de propagation. Pour des applications telles que le pompage optique de lasers à gaz, la connaissance de la dynamique du plasma est fondamentale car elle influence, par exemple, le maintien de l'inversion de population dans le milieu actif.

L'étude expérimentale des phénomènes hydrodynamiques produits par la décharge de surface a été réalisée au moyen d'une caméra à convertisseur d'image (CCD) intensifiée (caméra PCO, modèle Dicam-2). Le temps d'ouverture de la caméra est au minimum de 5 ns et sa sensibilité spectrale est limitée au domaine du visible par la configuration expérimentale retenue (objectif en verre, interface caméra-cellule dans l'air). 
La figure 3 montre une séquence de trois photographies transversales du plasma ; elles sont effectuées respectivement à 5,7 et $10 \mu$ s après le début d'une décharge de $15 \mathrm{kV}$ dans 1 bar d'argon. Le temps d'exposition est de 80 ns et la résolution spatiale de 0,1 $\mathrm{mm}$. Le plasma de forme demi-cylindrique est précédé par un front lumineux de même forme. Ce front correspond à la lumière émise par le plasma et diffusée par la couche de gaz comprimé due à la propagation d'une ou plusieurs ondes de choc. Celles-ci sont formées par l'injection rapide d'une forte énergie dans un volume restreint. Leur nombre est lié à l'homogénéité de la résistivité le long du canal conducteur; les ondes sont d'autant plus nombreuses que l'homogénéité du canal est faible. La zone sombre, visible au centre du plasma, correspond à l'ombre de la cathode située dans le champ visuel de la caméra.

La figure 4 permet de suivre l'évolution spatio-temporelle du front du plasma et de l'onde de choc associée.

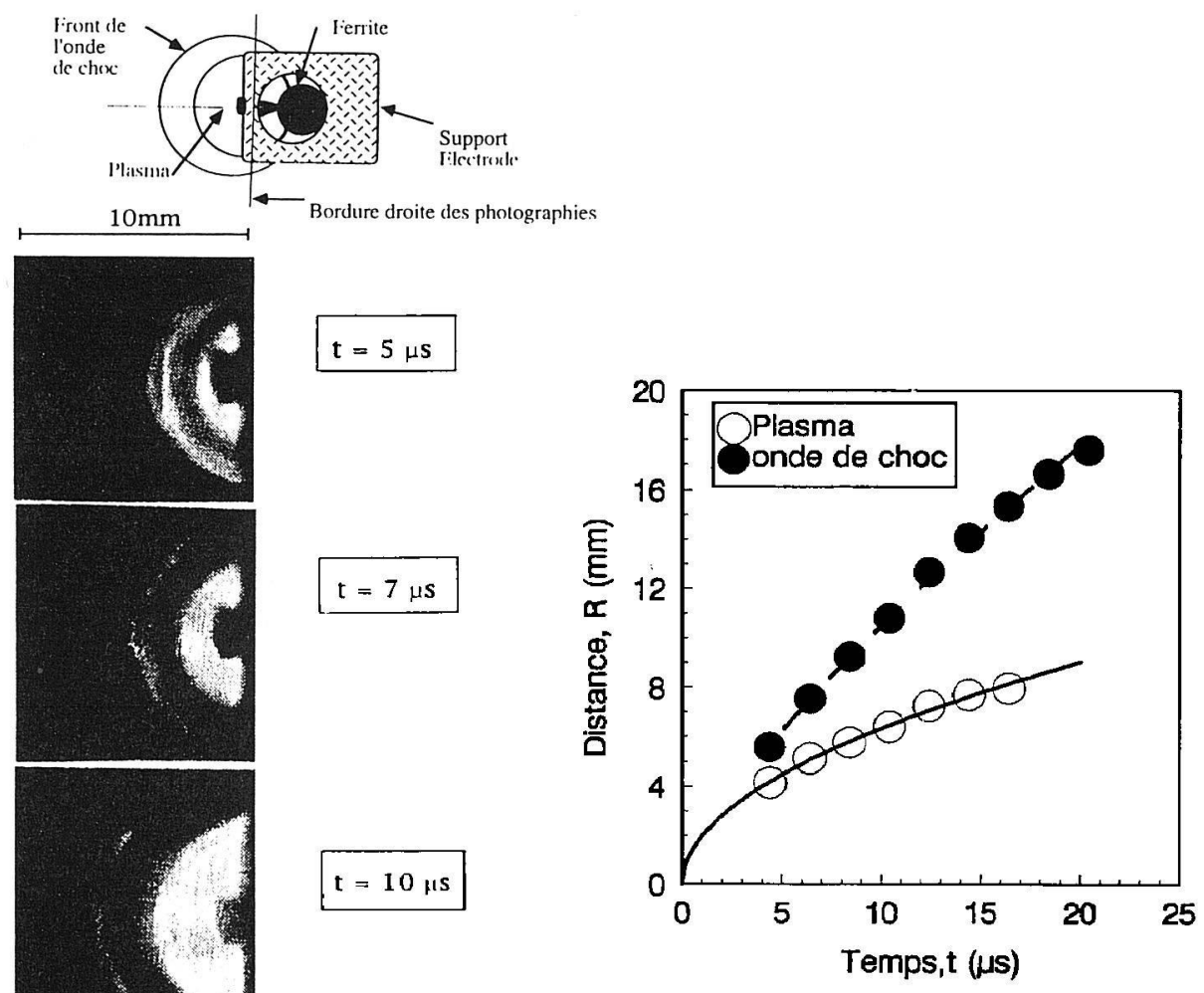

Fig. 4 : Evolution temporelle des fronts du plasma et de l'onde de choc.

Fig. 3 : Evolution temporelle des phénomènes dynamiques produits par une décharge de $15 \mathrm{kV}$ dans un bar d'argon. 
Chaque couple de points expérimentaux (front du plasma : $\bigcirc$, onde de choc : $\bullet$ ) est obtenu à partir d'une photographie instantanée $(4 \mu \mathrm{s}<\mathrm{t}<20 \mu \mathrm{s}, \Delta \mathrm{t}=80 \mathrm{~ns})$. Le phénomène étudié est parfaitement reproductible d'une décharge à l'autre pour des conditions expérimentales identiques. Pour une décharge de $12 \mathrm{kV}$ dans 1 bar d'argon, le plasma évolue dans une zone de rayon égal à $0,5 \mathrm{~mm}$, avec une vitesse maximale de 700 $\mathrm{m} / \mathrm{s}$ à $8 \mu \mathrm{s}$, diminuant ensuite jusqu'à $200 \mathrm{~m} / \mathrm{s}$ pour $\mathrm{t}=18 \mu \mathrm{s}$. L'onde de choc, se propage beaucoup plus loin et rapidement avec une vitesse de $1500 \mathrm{~m} / \mathrm{s}$ à $t=8 \mu \mathrm{s}$. Les courbes qui figurent sur le graphe (Fig. 4) correspondent à des fonctions du type $\mathrm{R}=a \mathrm{t}^{n}$, où $a$ et $n$ sont deux réels.

S'appuyant sur les résultats d'études théoriques ${ }^{7-8}$, l'étude expérimentale de la variation spatio-temporelle de l'onde de choc a été menée en fonction de l'énergie électrique $(E)$ injectée lors de la décharge $(100 \mathrm{~J}<\mathrm{E}<800 \mathrm{~J})$ et de la densité $\left(\rho_{0}\right)$ du gaz environnant $\left(0,8 \mathrm{~g} / 1<\rho_{0}<2,5 \mathrm{~g} / \mathrm{l}\right)$. Il est ainsi possible d'établir à partir des résultats expérimentaux la relation suivante $: \mathrm{R}=\mathrm{k}\left(\mathrm{E} / \rho_{0}\right)^{n / 2}{ }_{\mathrm{t}} n$, avec $\mathrm{k} \approx 0,32$ et $n \approx 0,75, \mathrm{R}$ étant en $\mathrm{mm}, \mathrm{t}$ en $\mu \mathrm{s}, \mathrm{E}$ en Joule et $\rho_{0}$ en $\mathrm{g} / \mathrm{l}$.

Cette relation est très proche de celle établie par Zel'dovich permettant de décrire l'expansion d'une onde de détonation. Seul le coefficient $n$ diffère, avec $n=0,4$ dans la formule de Zel'dovich ${ }^{7}$. Nous attribuons cette différence à la forme de l'injection spatiotemporelle de l'énergie. Dans le cas de Zel'dovich, l'injection de l'énergie est supposée ponctuelle et instantanée alors que dans notre configuration expérimentale l'injection se fait le long d'une ligne (canal conducteur), et dure pendant plus de $5 \mu \mathrm{s}$ (cf. Fig. 2 ). Enfin une partie de l'énergie stockée est injectée dans le canal, l'autre l'étant directement dans le gaz. Dabora 8 a développé un modèle plus proche de nos conditions expérimentales dans lequel l'énergie déposée s'exprime sous la forme $E=W t^{\beta}$, le coefficient $W$ représentant l'énergie injectée par unité de longueur, de surface ou de volume. Cependant cette approche n'est valable que pour la durée de l'injection d'énergie $(0<t<6 \mu \mathrm{s})$ et, durant la majeure partie de cette période, les moyens de diagnostics mis en oeuvre (caméra $\mathrm{CCD}$ ) n'ont pas permis la détection de l'onde de choc qui est alors confondue avec le plasma.

\section{ETUDE SPECTROSCOPIQUE}

Une étude spectroscopique de l'émission lumineuse du plasma a été entreprise dans un domaine spectral compris entre $115 \mathrm{~nm}$ et $220 \mathrm{~nm}$. L'émission est mesurée à l'aide d'un spectromètre. Il est constitué d'un monochromateur VUV (ARC VM502, réseau de 1200 traits $/ \mathrm{mm}$ ) de $0,2 \mathrm{~m}$ de longueur focale et d'un analyseur optique multicanaux (OMA) (PI IVUV 700). Le spectromètre est relié à l'enceinte par un tube de $0,5 \mathrm{~m}$ de long et de $25 \mathrm{~mm}$ de diamètre dans lequel une pression résiduelle inférieure à $10^{-4}$ Torr est maintenue. L'enceinte et l'ensemble spectromètre-tube sont séparés l'un de l'autre par une fenêtre en $\mathrm{MgF}_{2}$. La largeur de la fente d'entrée est de $25 \mu \mathrm{m}$, ce qui correspond à une résolution de $0,2 \mathrm{~nm}$. L'étalonnage en longueur d'onde est réalisé à l'aide d'une lampe au deutérium (Hamamastu, L 879-01). Les longueurs d'onde sont déterminées ainsi avec une valeur absolue de $\pm 0,5 \mathrm{~nm}$ dans lintervalle de longueurs considéré. Dans la plupart des expériences, les spectres ont été acquis avec une durée d'ouverture de l'OMA de $200 \mu \mathrm{s}$. Pour l'étude de l'évolution temporelle, la durée d'ouverture a été fixée à 200 ns et retardée de 0,6 à $1000 \mu$ s par rapport à l'instant initial de la décharge ( $t=0$ lorsque la tension s'écroule). L'intensité des spectres présentés ciaprès n'a pas été corrigée en fonction des caractéristiques optiques de la chaîne d'acquisition. 
La figure 5 montre trois spectres d'émission obtenus à partir d'une décharge de 12 $\mathrm{kV}$ dans 1 bar d'argon ou d'hélium et pour une pression résiduelle de $10^{-4}$ Torr. En l'absence de gaz, le spectre obtenu est principalement composé de raies intenses, dues à

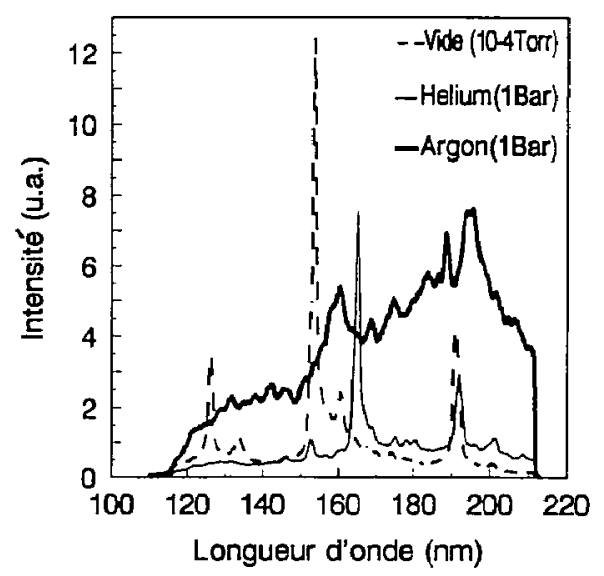

Fig. 5 : Spectres des décharges de surface sur ferrite pour une tension de $12 \mathrm{kV}\left(10^{-4}\right.$ Torr, argon et hélium (l bar)).

des transitions électroniques d'atomes ionisés provenant du canal conducteur situé sur la surface de la ferrite (Ni II, Fe III, Mn IV, ...) ou encore des électrodes. La détermination précise de la contribution d'un élément à une raie d'émission donnée est impossible du fait que les éléments constituants la ferrite présentent des émissions très proches les unes des autres dans le domaine spectral considéré et que la résolution en longueur d'onde de la chaîne de mesure est insuffisante. Toutefois, on observe une prédominance de l'émission liée au fer, constituant majoritaire du canal conducteur $(>80 \%)$.

Le spectre de la décharge dans l'hélium est dominé par de fortes raies d'émission et un faible continuum. Cependant, l'intensité des lignes dues aux éléments de la ferrite est maintenant atténuée et on observe l'apparition d'une raie intense à 164,3 nm de He II. Dans le cas de l'argon, le spectre est marqué par la présence d'un fort continuum. Celui-ci est principalement dû aux processus de recombinaison et au rayonnement de freinage. L'émission d'un continuum plus intense dans le cas d'une décharge dans l'argon plutôt que dans l'hélium s'explique par la différence entre les valeurs des potentiels d'ionisation de ces deux gaz : $24,58 \mathrm{eV}$ pour He et $15,76 \mathrm{eV}$ pour Ar. En effet, les atomes d'argon s'ionisant plus facilement par collision avec les éléments issus du canal conducteur ou sous l'effet de la décharge électrique elle même, il en résulte un taux de recombinaison plus important que dans l'hélium.

Une étude de l'évolution temporelle des spectres pour des décharges réalisées dans de l'argon ou de l'hélium à une pression de un bar, a également été effectuée. La figure 6 montre les résultats obtenus pour une décharge de $12 \mathrm{kV}$. Dans le cas de l'argon (Fig. 6a), l'allure générale du spectre est indépendante du temps, seule l'intensité varie. Dans le domaine spectral considéré (115 - 175 nm), elle suit l'évolution temporelle de la puissance injectée, et atteint sa valeur maximale à $6,6 \mu \mathrm{s}$. Cet instant correspond au 
maximum de la puissance électrique déposée. Les décharges dans l'hélium (Fig. 6b) ont une évolution temporelle spectrale quasiment identique, si l'on excepte l'apparition d'un pic à $164,3 \mathrm{mn}$. Ce pic, dû à He II, a une forte intensité uniquement dans l'intervalle de temps où la puissance électrique injectée est proche de sa valeur maximale. Les particules éjectées de la ferrite ont alors suffisament d'énergie pour ioniser l'hélium qui est également ionisé directement par la décharge électrique.

Enfin une étude de l'évolution de l'émission spectrale en fonction de la tension de charge (12 à $22 \mathrm{kV})$ a été effectuée pour des décharges de surface dans un bar d'hélium ou d'argon. On observe une augmentation des intensités des raies ainsi que celle des continua. Cette croissance évolue proportionnellement au carré de la tension de charge dans le cas de l'argon, alors qu'un phénomène de saturation apparaît dans l'hélium pour une tension de charge située vers $18 \mathrm{kV}$. Dans ce dernier cas, on s'aperçoit que lorsque la tension de charge augmente, les signaux électriques de courant et tension oscillent de plus en plus, avec un faible amortissement. Ce comportement s'explique par une mauvaise adaptation d'impédance entre le circuit d'excitation électrique et le plasma, entraînant une diminution du rendement d'énergie électrique injectée d'où l'apparition de ce phénomène de saturation.

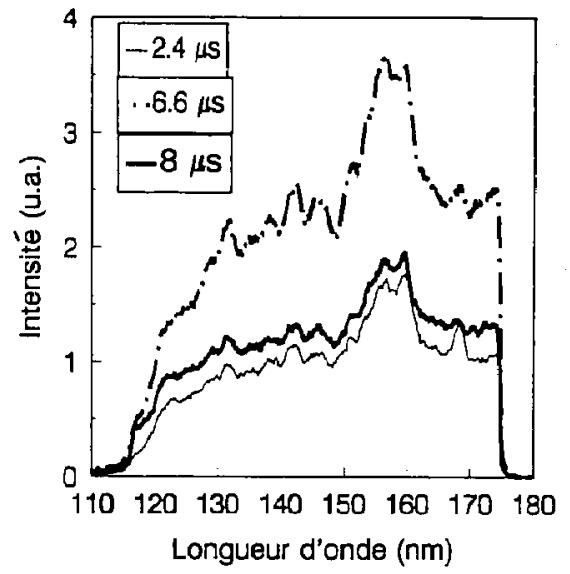

(a)

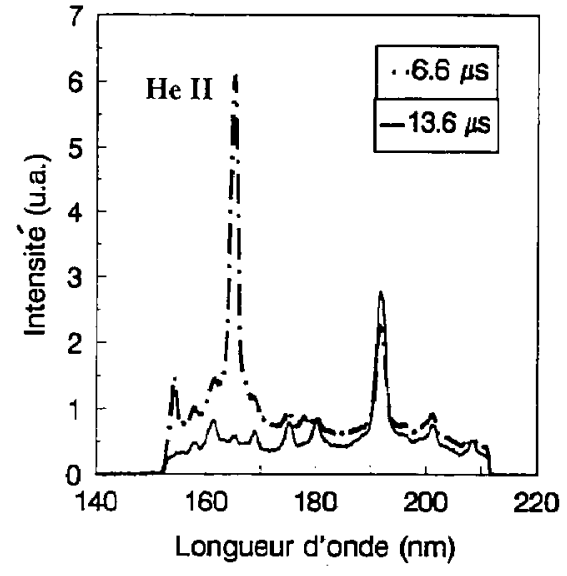

(b)

Fig. 6 : Evolution temporelle des spectres de décharges sur ferrites dans un bar d'argon (a) et un bar d'hélium (b). La tension de charge est de $12 \mathrm{kV}$.

\section{CONCLUSION}

Les décharges électriques sur ferrites formées $\left(\mathrm{Ni}^{-} \mathrm{Zn} \mathrm{Fe} \mathrm{O}_{4}\right)$ permettent la création de plasmas de forte intensité lumineuse dans les domaines de l'ultraviolet et de l'ultraviolet du vide. Cette source VUV impusionnelle s'obtient dans des conditions raisonnables de tension de décharge et de pression de gaz avec la possibilité de fonctionner en mode répétitif rapide $(\sim 100 \mathrm{~Hz})$. L'étude expérimentale de l'hydrodynamique du plasma réalisée à l'aide d'une caméra rapide, a permis de mettre en 
évidence la formation d'une onde de choc suite à l'injection rapide (quelques $\mu \mathrm{s}$ ) d'une énergie intense $(200$ à $1000 \mathrm{~J})$ dans le canal conducteur de faible volume $\left(100 \mu \mathrm{m}^{2} \times 18\right.$ $\mathrm{cm})$. Le plasma de forme demi-cylindrique reste confiné dans un rayon de $0,5 \mathrm{~cm}$ près de la surface de la ferrite. L'onde de choc se propage radialement suivant une loi du type $R$ $=\mathrm{k}\left(\mathrm{E} / \rho_{0}\right)^{n / 2} \mathrm{t}^{n}$, comme dans le cas d'une onde de détonation.

L'étude des propriétés spectroscopiques des décharges sur ferrites dans un domaine compris entre 115 et $220 \mathrm{~nm}$ a montré la présence de raies d'émissions attribuées aux transitions électroniques des espèces ionisées de la ferrite et ayant été éjectées du canal conducteur au cours de la décharge. La présence d'un gaz environnant se manifeste de manière différente selon la nature du gaz : on peut observer des raies supplémentaires caractéristiques du gaz ionisé (cas de l'hélium) ou bien un continuum de recombinaison se superposant aux raies (cas de l'argon).

Une caractéristique également attractive des décharges de surface sur ferrites formées est la possibilité de créer des sources VUV de grande surface émettrice. Ainsi, une source VUV d'une longueur de $80 \mathrm{~cm}$ pour le pompage optique de lasers a été ainsi réalisée avec une tension de charge de $20 \mathrm{kV}$.

\section{Remerciements :}

Ces travaux n'auraient été possibles sans le soutien financier du groupe 4 de la DRET, et le soutien scientifique de tous les membres du groupe Nouveaux Lasers de I'IMFM.

\section{Bibliographie :}

[1] Watanabe K., Kashiwabara S., and Fujimoto R., Appl. Phys. Lett. 50, (1987) 629.

[2] Sentis M., Chazaud F., Delaporte Ph., Marine W., Appl. Phys. Lett. 63, (1993) 2333.

[3] Watanabe K., Furusho K., Kashiwabra S. and Fujimoto R., J. Appl. Phys. 68, (1990) 5059 .

[4] Watanabe K, van Goor F.A., Fujimoto R. and Witteman W.J., GCL - Seventh International Symposium on Gas Flow and Chemical Lasers, SPIE 1031,(1988) 450.

[5] Kogelschatz U., Applied Surface Science 54, (1992) 410.

[6] Sentis M.L., Granier Ph., Chazaud F., Marine W.I., Gerry M. and Sze R.C., Appl. Surf. Science 68, (1993) 185.

[7] Zel'dovich Ya.B. and Raiser Yu., Physics of Shock Waves and High Temperature Hydrodynamic Phenomena, (Academic Press, New York, 1966).

[8] Dabora E.K., AIAA Journal 10 (1972). 